\title{
Model Smoke Stream Adsorption over Cellulose Acetate Stick with Three-dimensional Temperature Gradient by Combining in-situ DRIFTS with Infrared Thermal Imaging
}

\section{Xin Xu}

China Tobacco Yunnan Industrial Co Ltd

Xi Du

Eastman Shuangwei Fibers Company Ltd

Feng Zheng

Anhui Tobacco Industrial Co Ltd

Lisheng Guo

Anhui University

Panwei Shi

Eastman Shuangwei Fibers Company Ltd

Mengmeng $\mathbf{L i}$

Anhui University

Zhengyu Fang

Anhui University

Jiuyi Liu

Anhui University

Mengdie Cai ( $\square$ caimengdie1987@163.com )

Anhui University https://orcid.org/0000-0002-7605-4895

Song Sun

Anhui University

\section{Research Article}

Keywords: Cellulose Acetate Stick, in -situ, DRIFTS, CO, Smoke Stream, Temperature Distribution, 3D Adsorption Model

Posted Date: September 20th, 2021

DOI: https://doi.org/10.21203/rs.3.rs-883660/v1 
License: (c) (i) This work is licensed under a Creative Commons Attribution 4.0 International License. Read Full License

Version of Record: A version of this preprint was published at Cellulose on January 10th, 2022. See the published version at https://doi.org/10.1007/s10570-022-04415-x. 


\section{Model Smoke Stream Adsorption over Cellulose Acetate Stick with Three-dimensional Temperature Gradient by Combining in-situ DRIFTS with Infrared Thermal Imaging}

Xin Xu $\bullet$ Xi Du $\bullet$ Feng Zheng $\bullet$ Lisheng Guo $•$ Panwei Shi $\bullet$ Mengmeng Li $\bullet$ Zhengyu

Fang • Jiuyi Liu $\bullet$ Mengdie Cai $\bullet$ Song Sun

$\mathrm{Xin} \mathrm{Xu} \cdot$ Feng Zheng

Anhui Tobacco Industrial Co., Ltd, Hefei, 230088, China

Lisheng Guo • Mengmeng Li • Zhengyu Fang • Jiuyi Liu • Mengdie Cai $(\bowtie) \cdot$ Song Sun

School of Chemistry and Chemical Engineering, Anhui University, Hefei, Anhui

230601, China

E-mail: caimengdie1987@163.com (M. Cai)

Xi Du • Panwei Shi $(\bowtie)$

Eastman Shuangwei Fibers Company Limited, Hefei, Anhui 230601, China

E-mail: shipw@esfcl.com (P. Shi) 
$1 \quad$ Abstract

2 Understanding the adsorption of the smoke stream (SR) on cellulose acetate stick as

3 cigarette filter with different temperatures is beneficial for controlling chemical

4 emissions and reducing the toxic effect of smoking on human health. However the

5 investigation of corresponding adsorption properties was missing because the

6 adsorption of smoke stream (SR) on cellulose acetate stick is sensitive with the three-

7 dimensional temperature gradient. In this work, the adsorption of typical smoke stream

8 substances, such as CO, propylene glycol, formaldehyde, and acetone, on cellulose

9 acetate stick were studied by in-situ diffuse reflectance Fourier transform infrared

10 spectroscopy with different temperatures assisted by the infrared thermal imaging

11 method. The adsorption capacities of cellulose acetate stick to these typical smoke

12 stream substances is dependent on the adsorption time and temperature. The adsorption

13 properties all fitted well with the Freundlich model. By a spectroscopic and

14 mathematical explanation, quantifying contours of adsorption was performed. The 3D

15 model of the normalized $\mathrm{CO}$ adsorption of cellulose acetate stick versus the spatial

16 coordinates and time was established. This study gives unparalleled insight into

17 smoking release characteristics of tobacco filtered by cellulose acetate and regulating

18 cellulose acetate stick for reducing the negative effect of smoke on human health.

19 Keywords: Cellulose Acetate Stick $\cdot$ in-situ DRIFTS $\cdot$ CO $\cdot$ Smoke Stream $\cdot$ Temperature

Distribution $\cdot 3 D$ Adsorption Model. 


\section{Introduction}

Cigarette smoke is a complex mixture produced by tobacco combustion, cracking and distillation (Boue et al., 2020). CO and low-carbon aldehydes and ketones are toxic components of cigarette smoke stream, seriously affecting human health (Pasupathi et al., 2009; Starek and Podolak, 2009). Over the years, a number of studies have investigated the harmful effect of tobacco and smoke on human health (Adam et al., 2006; Ma, 2021). The release of CO and low-carbon aldehydes and ketones is an important index for measuring the combustion and pyrolysis characteristics and smoke toxicity of tobacco, as well as for reflecting the comprehensive quality of cigarettes (Feng et al., 2013). The release of CO and low-carbon aldehydes and ketones is greatly affected by the combustion and pyrolysis environment (Pauwels et al., 2018). Therefore, the investigation of their adsorption and retention characteristics at different temperatures is beneficial for guiding the design and development of filtrational materials for cigarettes, and the optimization of cigarette filter, comprehensively improving the sensory quality and safety of cigarette smoke.

At present, the main cigarette filtrational materials include cellulose acetate, polypropylene, and polyethylene terephthalate, etc.(Chau et al., 2020; Dong et al., 2021; Zhang et al., 2020). Among them, cellulose acetate stick as the cigarette filter can balance the smoke substances by trapping the excessive concentration of harmful cigarette smoke (Dwyer and Abel, 2015). At the same time, it exerts a filtering role, intercepting smoke powder particles and adjusting suction resistance (Du et al., 2015). Previous studies investigated the overall transfer of CO, 1, 2-propanediol, glycerol, 
nicotine and some flavor components in different heated cigarette materials, aerosols and filters, and the change of the smoking cycle (puffing-smoldering-puffing) release of main components of smoke substances ( $\mathrm{Li}$ et al., 2018; Li et al., 2015). Some research groups have also studied the smoke substances release law of cigarette filter material and length on the main components of smoke substances under different heat sources, smoke core sections and suction conditions (Fei et al., 2014).

However, the change law of the adsorption structure and adsorption capacity of cigarette smoke component chemistry on the surface of cellulose acetate stick with temperature/ coordinate axes is challenging, because the adsorption of $\mathrm{CO}$ and lowcarbon aldehydes and ketones on the filter material is sensitive to the three-dimensional temperature gradient which is difficult to obtain on the cigarette filter stick.

In-situ infrared spectroscopy is useful non-invasive and real-time device for evaluating changes in a chemical structure and monitoring the changes of characteristic functional groups with temperature (Meunier, 2016; Proaño et al., 2019; Shi et al., 2020; Zhong et al., 2020). Among the available characterization modes of infrared spectroscopy, diffuse reflectance Fourier transform infrared spectroscopy (DRIFTS) mode is the most suitable for in-situ characterization for gas-solid interface reaction, as it can collect and analyze the surface and interface information carried by the electromagnetic radiation reflected from the surface (Dzara et al., 2019; Li et al., 2020; Tofan-Lazar et al., 2013). Yet, for the measurement of the three-dimensional temperature gradient of the cigarette filter stick, the surface contact between the measuring instrument and the measured object can disturb the original temperature field 
of the object, affecting the transmission of the temperature sensing element, and leading to the inaccurate output signal (Li et al., 2014; Li et al., 2016). Fortunately, the infrared thermal imaging technology does not interfere with the temperature of the measured object without inertial effect in the transmission of optical information, which can smoothly change the path and ensure the integrity of data (Jia et al., 2017; Pham Xuan et al., 2021; Roehl et al., 2009).

In this study, we used infrared thermal imaging technology to detect the threedimensional temperature distribution information of cellulose acetate stick of burning cigarette. Based on this, we used in-situ DRIFTS to characterize the adsorption of CO, propylene glycol, and typical low-carbon aldehydes and ketones, including formaldehyde and acetone, on the surface of cellulose acetate sticks at the above temperature range of the cellulose acetate stick. The corresponding adsorption kinetic model were established. This work may provide a new perspective for guiding the design and development of filter stick materials for cigarettes and improving the suction safety of filters.

\section{Experimental}

\section{Materials and Reagents}

The chemicals used in the experiments, including formaldehyde solution, acetone, and propylene glycol were purchased from Sinopharm Chemical Reagent Co., Ltd. Deionized water (resistivity of 18.2 M 2 ) was purified by using a Millipore system. Gases used in the study were Argon (Ar, 99.99+\%) and the compressed air (20 vol.\% 
$\left.\mathrm{O}_{2} / \mathrm{N}_{2}\right)$.

The cellulose acetate sticks used in this study were obtained from Eastman Shuangwei Fibers Company Limited. The degree of acetyl substitution of cellulose acetate is between 2.0 and 2.7. Its molecular formula is $\left[\mathrm{C}_{6} \mathrm{H}_{7} \mathrm{O}_{2}\left(\mathrm{OCH}_{3}\right)_{x}(\mathrm{OH})_{3-x}\right], \mathrm{n}=$ 200-400). It is widely used as cigarette filter tow (cigarette cellulose acetate tow) in commercial with a radius of $4 \mathrm{~mm}$, and a length of $20 \mathrm{~mm}$.

\section{Characterization of samples}

The morphology of the samples were investigated by a scanning electron microscope (Sirion 200). CO-TPD was performed on AutoChem II 2920. Approximately $80 \mathrm{mg}$ of the sample was loaded into a U-type quartz tube, which was mounted on the instrument. Then the sample was pretreated in $\mathrm{Ar}$ at $100{ }^{\circ} \mathrm{C}$ for $0.5 \mathrm{~h}$ (heating rate $10^{\circ} \mathrm{C} / \mathrm{min}$ ). After cooling to room temperature, 5\% $\mathrm{CO}$ in $\mathrm{Ar}$ was passed through the sample for $1 \mathrm{~h}$, with subsequent flushing with helium at $100{ }^{\circ} \mathrm{C}$ for $1 \mathrm{~h}$. The TPD analysis was carried out in flowing $\mathrm{Ar}$ from $50{ }^{\circ} \mathrm{C}$ to $300{ }^{\circ} \mathrm{C}$ at a heating rate of $10{ }^{\circ} \mathrm{C} / \mathrm{min}$.

\section{Infrared thermal imaging test}

The three-dimensional temperature gradient of the filter stick during cigarette burning was observed and recorded by the Forward Looking Infrared (FLIR) thermal imaging system with a THERMACAM 25 camera and the THERMACAM Reporter 2000 software. The distance between the camera and the object was fixed from $10 \mathrm{~cm}$ to $50 \mathrm{~cm}$.

\section{in-situ DRIFTS measurements}



and ketones, in-situ DRIFTS measurements were performed on a Bruker IFS 66v/s FTIR spectrometer equipped with a self-built setup and a DRIFTS cell. As shown in Figure 1, the setup consists of a detection system, a reaction system, and a coupling reaction gas-dosing system. In the gas-dosing system, mass flow controllers were used to control the $20 \mathrm{vol} . \% \mathrm{O}_{2} / \mathrm{N}_{2}$ compressed air which carried $\mathrm{SR}$ vapor from the saturator containing typical low-carbon aldehydes and ketones (LCA) or propylene glycol (PG), such as formaldehyde solution, acetone, and propylene glycol. The water vapor was supplied and regulated to the cell via a by-pass line. The relative humidity in the cell was determined using an electronic hygrometer fixed in the by-pass line. The reaction system consists of a praying mantis DRIFTS accessory (Harrick Scientific) and a reaction cell (HVC, Harrick Scientific). The reaction cell is equipped with a sample cup with retaining plates in it and covered by a dome fitted with three windows (Figure S1).

121 Cooling water was circulated through a coil surrounding the base of the dome to 122 facilitate the reaction at room temperature.

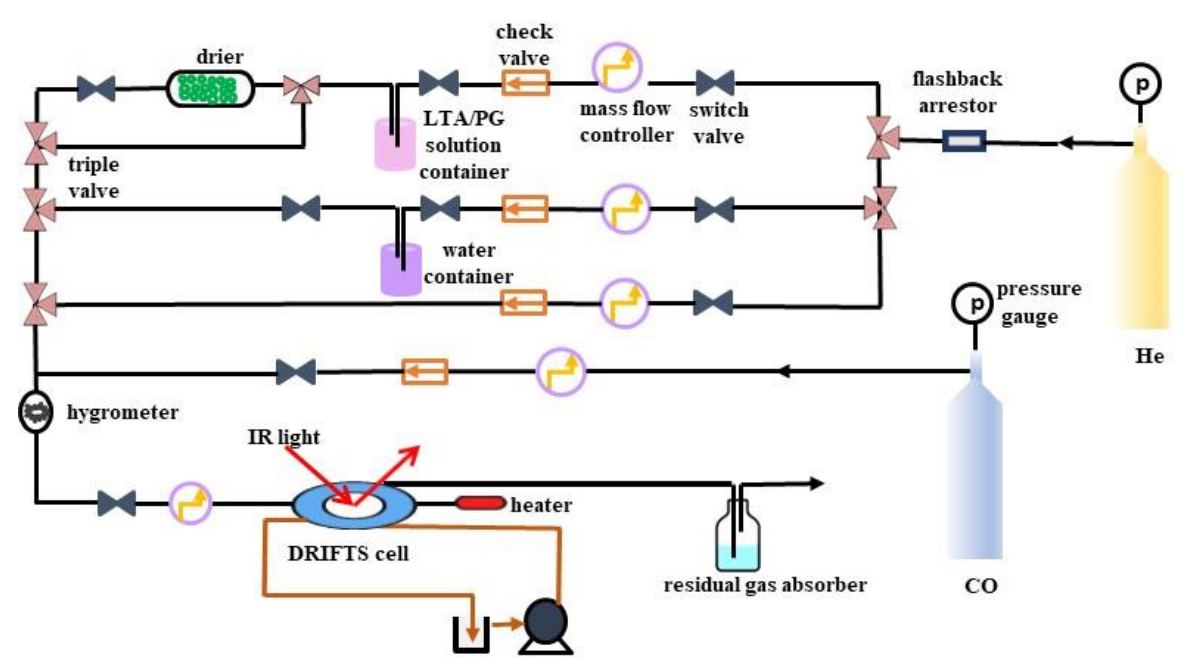

Figure 1. Schematic diagram of the experimental setup for in-situ DRIFTS measurements 


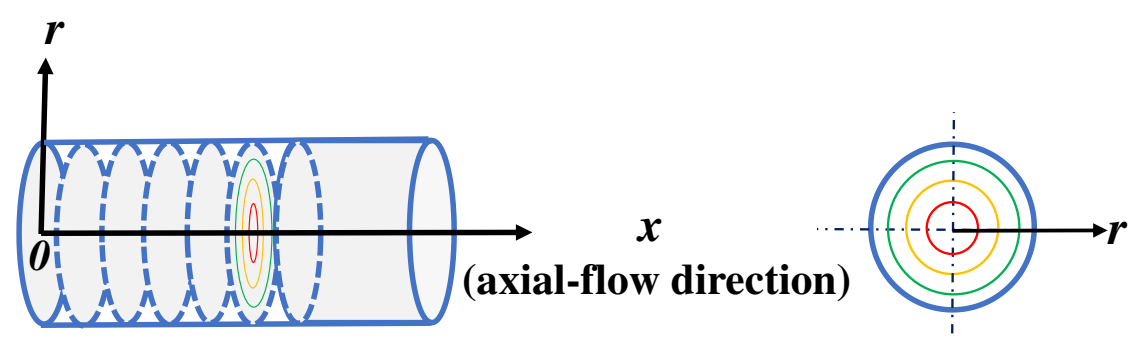

Figure 2. A schematic of cellulose acetate stick and its axial definition. $X$ is the axial direction: $0 \mathrm{~mm}$ corresponding to the lighting end of the cigarette. Different colors in the radial direction of the $2 \mathrm{D}$ cross section represent the temperature contours with different temperatures. important step in controlling the interception of smoke substances. The geometric model of cellulose acetate stick and its axial definition used in this study is shown in Figure 2. A cellulose acetate stick has a radius of $4 \mathrm{~mm}$, and a length of $20 \mathrm{~mm}$, and uniform agglomeration properties of three-dimensional space structure and towbonding structure (Figure 3).
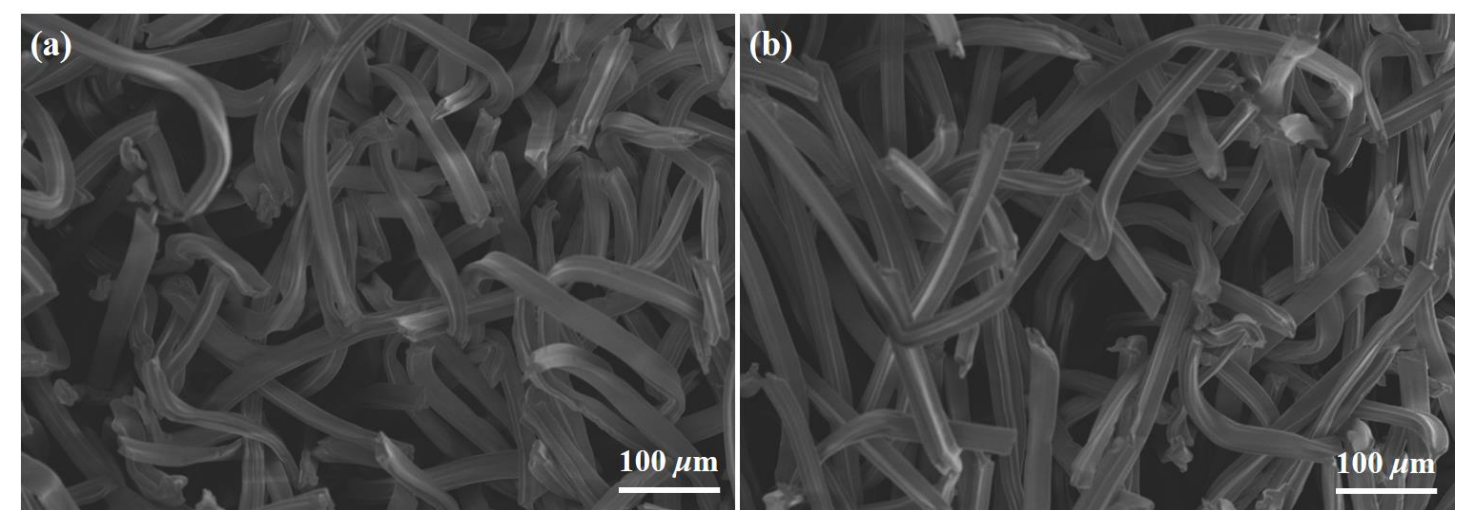

Figure 3. Cellulose acetate stick with the cross-section of axial-flow direction (a) and $r$ 
(SEM).

The temperature field in the cellulose acetate stick was detected by infrared thermal imaging. The FLIR camera based on the thermal imaging method was used to analyze the temperature distribution of cellulose acetate stick with cigarette burning. FLIR camera can detect minimum changes in temperature $(0.1$ degrees). The coordinate of the corresponding points in the 3D space can be obtained during temperature information acquisition according to the constructed 3D temperature model. Regarding temperature field simulation, the following condition for cellulose acetate stick were declared: 1) the porous medium is even and isotropic; 2) the porosity, specific heat capacity, density, and heat transfer coefficient are constant; 3) natural convection and radiation are ignored (Jiang et al., 2018).
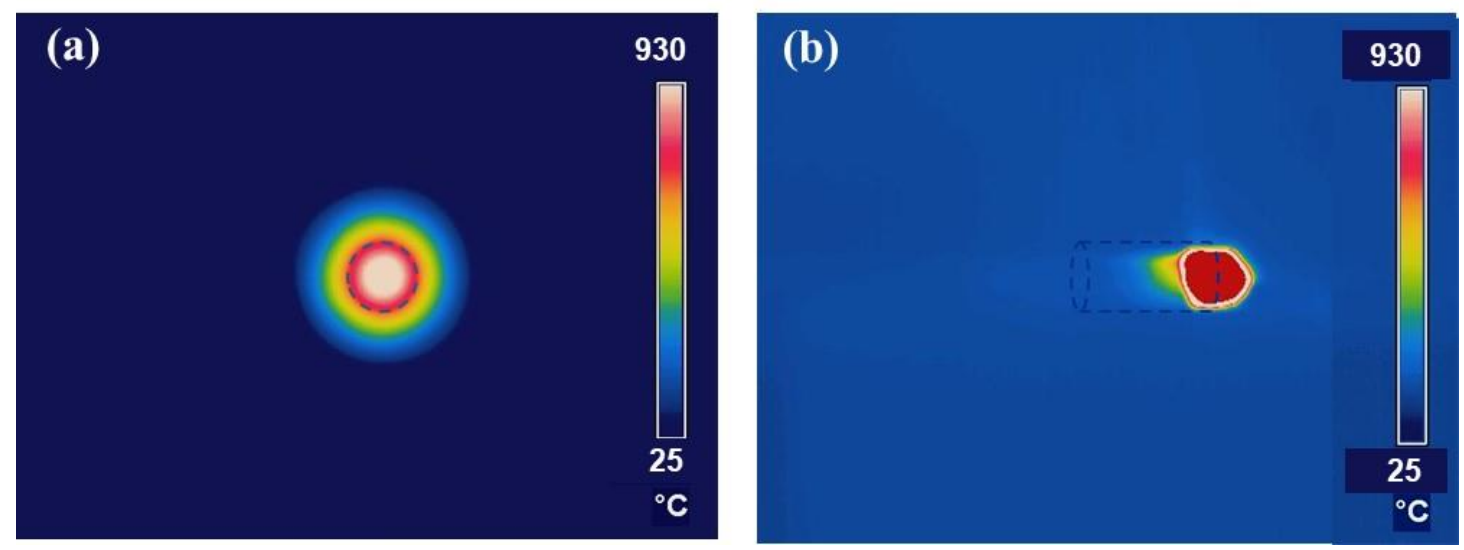

Figure 4. The infrared thermal images for a burning cigarette using cellulose acetate stick as a filter. The $2 \mathrm{D}$ cross-section temperature distribution at $x=0 \mathrm{~mm}$, the dotted circle represents the cross section of cellulose acetate stick (a); the temperature distribution of a cigarette along $\mathrm{x}$ axis, the dotted cylinder represents the cellulose acetate stick (b); which were recorded when the burning spot is almost close the 
(a)

(b)
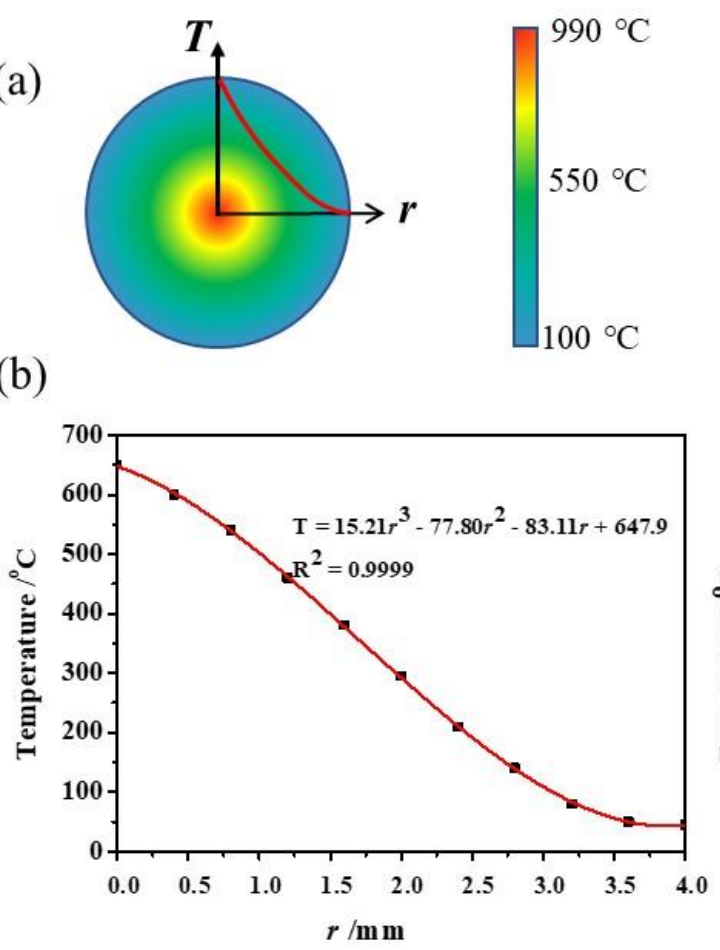
remained stable.

167

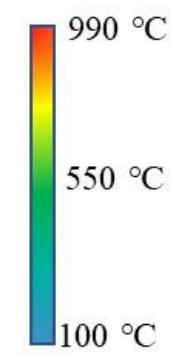

As shown in Figures 4a, the temperature field distribution of the 2D cross-section was axially symmetrical. When the burning spot was almost close to the cellulose acetate stick (Figure 4b), the highest temperature of the position in the cellulose acetate stick was $930{ }^{\circ} \mathrm{C}$ which is observed closest to the burning tobacco. The temperature gradually decreased from the core temperature of $930{ }^{\circ} \mathrm{C}$ to the edge temperature of $260{ }^{\circ} \mathrm{C}$ along the $r$-axis. The heat diffused along the $r$-axis and $x$-axis, and the temperatures distributions along the two axes were different. The core temperature from $930{ }^{\circ} \mathrm{C}$ at $x=0$ gradually decreased to $25{ }^{\circ} \mathrm{C}$ at $x=16 \mathrm{~mm}$ approximately, and then

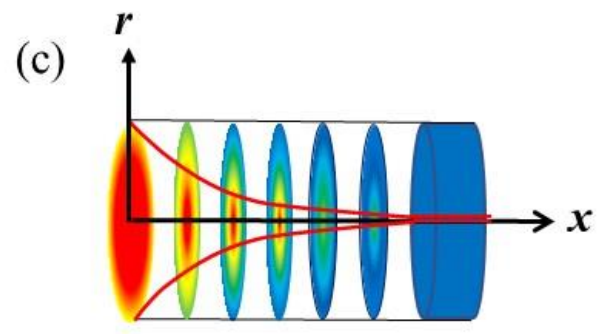

(d)

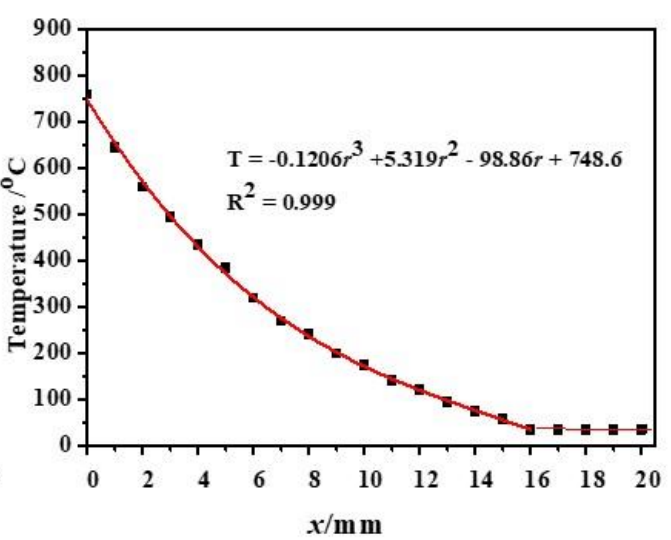

Figure 5. The radial temperature distribution of the cellulose acetate stick along with $r$ axis (a) with the fitting curve of radial temperature versus $r$ (b) at the cross-section of 
$170 x=10 \mathrm{~mm}$; The $2 \mathrm{D}$ cross-section average temperature distribution of the cellulose 171 acetate stick along the $x$-axis (c) with the fitting curve of temperature versus $x(\mathrm{~d})$; the 172 insets are the fitted polynomial equations.

From the infrared thermal images results, we obtained 3D temperature distribution

174 information with the fitting curves in the $r$-axis and $x$-axis (Figures $5 \mathrm{a}$ and $5 \mathrm{~b}$ ). The temperature distribution of the $2 \mathrm{D}$ cross-section under different $x$ coordinates was different. The highest temperature was always in the central region. The temperature gradually decreased along the radial direction. Take the $2 \mathrm{D}$ cross-section at $x=10 \mathrm{~mm}$ for example (Figure 5a), the curve of temperature versus $r$ coordinate (Figure 5b) can be fitted to a polynomial equation as follows.

$$
T=15.21 r^{3}-77.80 r^{2}-88.11 r+647.9 \quad \text { (Equation.1) }
$$

The fitted equations of temperature distribution for all the $2 \mathrm{D}$ cross-sections under different $x$ coordinates could be established in the same way as that at $x=10 \mathrm{~mm}$. Based on this, the average temperatures of all the cross-sections of the cellulose acetate stick could be easily acquired by the temperature integration of the $2 \mathrm{D}$ cross-section. The highest average temperature was observed at $x=0$, which is closest to the burning tobacco in the course of smoking; then the heat of $2 \mathrm{D}$ cross-section at $x=0$ diffuses along the $x$ axial-flow direction, and the temperature of 2D cross-section decreased to room temperature at $x \approx 16 \mathrm{~mm}$. The curve of the average temperature of $2 \mathrm{D}$ crosssection along the $x$-axis could also be calculated using a polynomial equation:

$$
T=0.0588 x^{3}-0.1603 x^{2}-48.18 x+613.55 \text { (Equation.2) }
$$

Which was shown in Figure 5d. The R-squares of the two equations were close to 
1.0, indicating the good predictability of the two fitted models. Additionally, the temperature gradients in the cellulose acetate stick were reduced both along the $x$ axial and radial directions. Through the acquisition and mathematical model analysis of the typical temperature data of cellulose acetate stick, the 3D temperature distribution model could be obtained. According to the 3D temperature distribution model, the temperature field of the whole cellulose acetate stick could be observed. On the other hand, we could easily get the temperature information at any point in the cellulose acetate stick from the obtained 3D temperature distribution model.

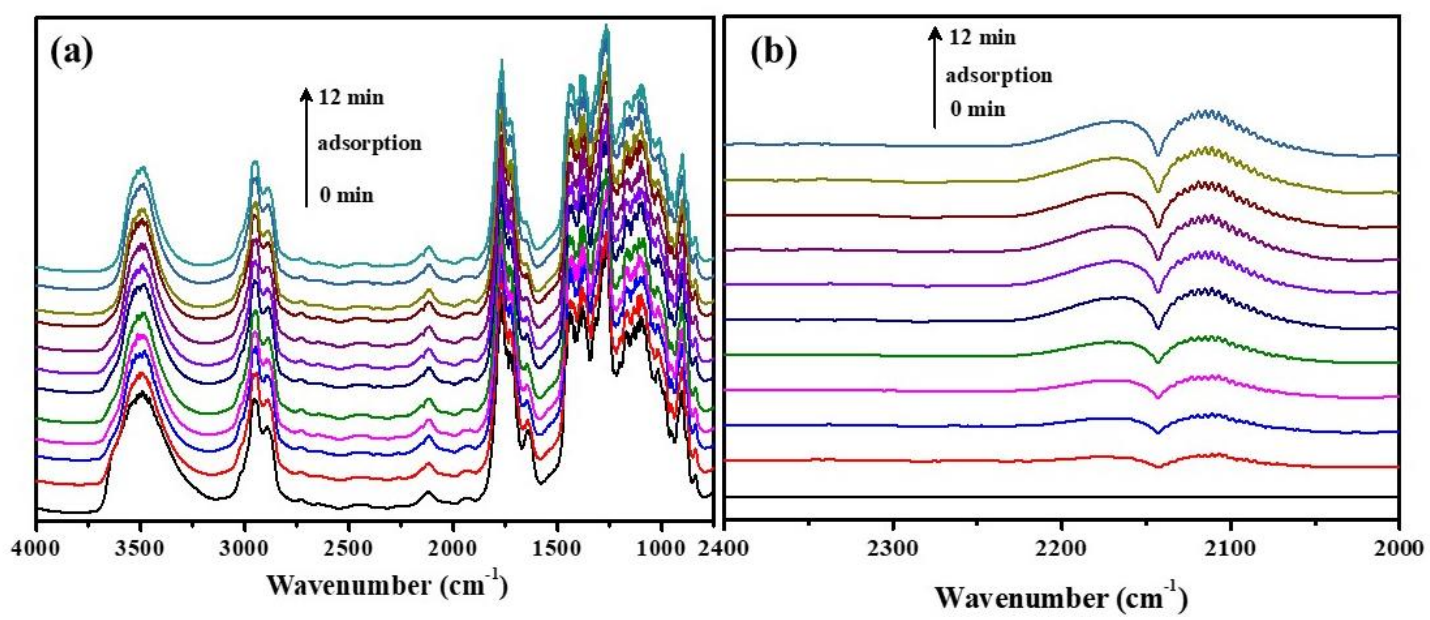

Figure 6. (a) In-situ DRIFTS spectra of dynamic adsorption of CO on cellulose acetate stick as a function of time in CO stream. (b) Differential spectra from (a). The condition temperature is $25^{\circ} \mathrm{C}$.

In-situ DRIFTS study of the surface-adsorbed species is powerful for investigating the adsorption kinetics. After determining the 3D temperature distribution of the cellulose acetate stick mentioned above, in-situ DRIFTS technique was used to test the adsorption characteristic of typical smoke substances, such as $\mathrm{CO}$, formaldehyde, 
acetone and propylene glycol, in the temperature range of $25-930{ }^{\circ} \mathrm{C}$. Firstly, as one of the main harmful substances in the smoke stream, $\mathrm{CO}$ was used as a probe molecule to test the adsorption characteristic of cellulose acetate stick at different adsorption temperatures for $12 \mathrm{~min}$. Figures 6 displays the variation of DRIFTS spectra of CO adsorption and the DRIFTS differential spectra of CO on cellulose acetate stick at room temperature. The band at $2130 \mathrm{~cm}^{-1}$ assigned to $\mathrm{CO}$ stretching $\left(v_{\mathrm{CO}}\right)$ indicated $\mathrm{CO}$

214 adsorption on cellulose acetate stick after exposure to CO (Wang et al., 2017). The 215 intensity of the band became stronger with the adsorption time until it reached $\mathrm{CO}$ 216 adsorption equilibrium at around $8 \mathrm{~min}$. The normalized $\mathrm{CO}$ adsorption amount remained unchanged after $8 \mathrm{~min}$.

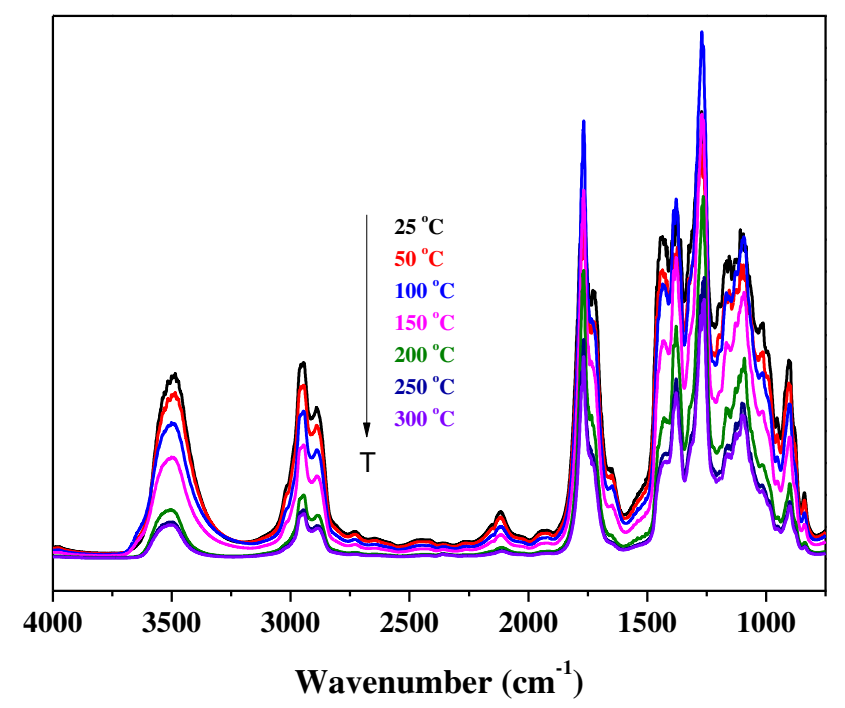

Figure 7. The in-situ DRIFTS spectra of CO versus adsorption temperature on cellulose acetate stick after $\mathrm{CO}$ adsorption equilibrium.

The in-situ DRIFTS spectra of CO adsorbed on the cellulose acetate stick surface

222 for reaching $\mathrm{CO}$ adsorption equilibrium in the temperature range from 25 to $300{ }^{\circ} \mathrm{C}$ 
were further organized, as shown in Figure 7. It can be seen that the CO saturated adsorption capacity decreased with the increase of adsorption temperature. When the adsorption temperature reached $300{ }^{\circ} \mathrm{C}$, the amount of adsorbed $\mathrm{CO}$ could be neglected.

Combined with the infrared thermal imaging results, we concluded that the $\mathrm{CO}$ adsorption occurred only in the partial region of cellulose acetate stick where the temperature was between 25 and $300{ }^{\circ} \mathrm{C}$, while, there was no $\mathrm{CO}$ adsorption in the region of cellulose acetate stick where the temperature was beyond $300{ }^{\circ} \mathrm{C}$.

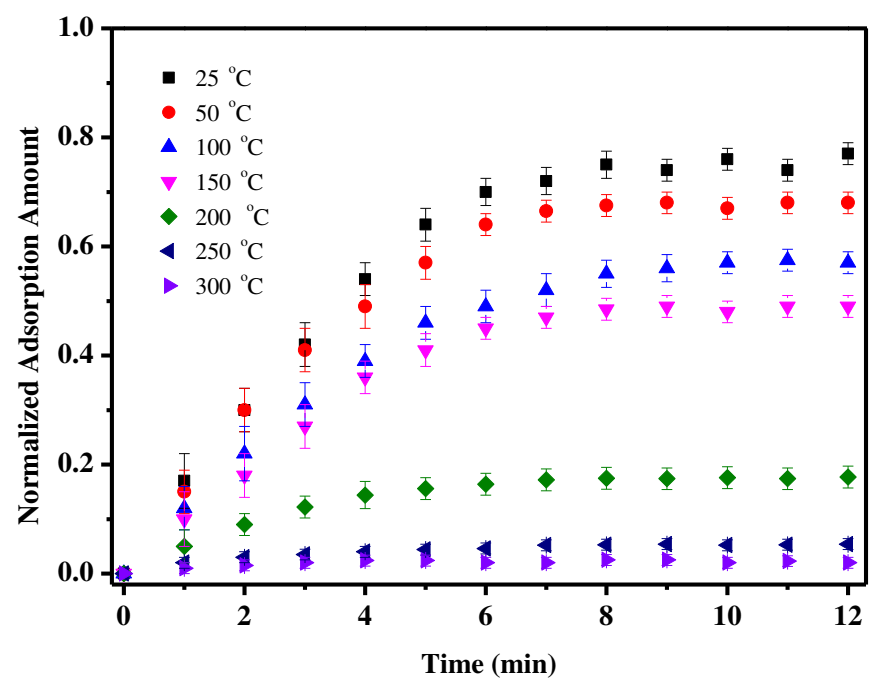

Figure 8. Breakthrough curves of $\mathrm{CO}$ adsorption versus adsorption temperature on cellulose acetate stick change of intensities of asymmetric and symmetric of $v_{\mathrm{C}-\mathrm{O}}$ reached a steady level after 8 min approximately. As the temperature is increasing from 25 to $300{ }^{\circ} \mathrm{C}$, the $\mathrm{CO}$ saturated adsorption amount gradually deceases, because the high temperature is not 

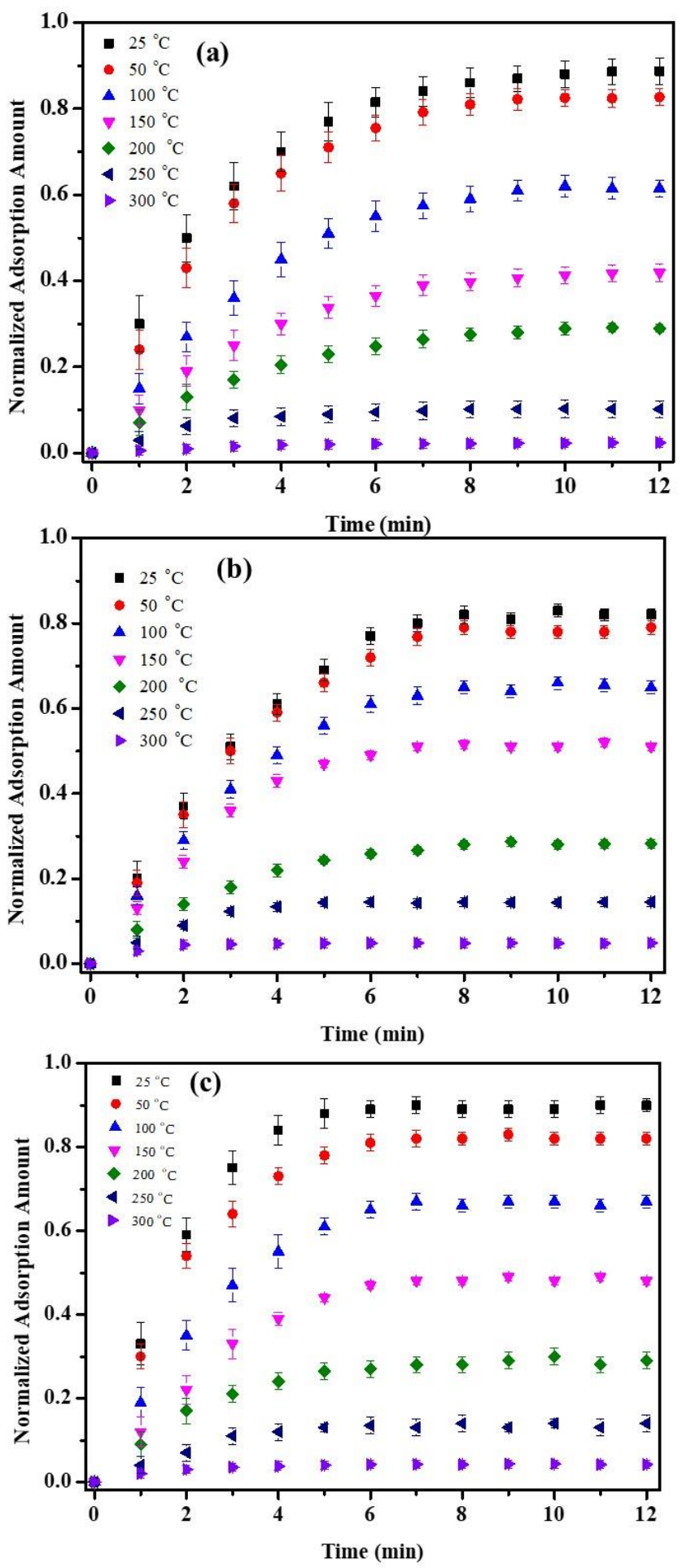

240 Figure 9. Breakthrough curves of formaldehyde (a), acetone (b) and propylene glycol adsorption (c) versus adsorption temperature on the cellulose acetate stick 

substances are also harmful to human health, and their adsorption ability on the surface of the cellulose acetate stick are also greatly affected by the adsorption temperature (Alalwan et al., 2020). The adsorption characteristic of such smoke stream substances on the surface of cellulose acetate stick were observed by in-situ DRIFTS using the same approach as $\mathrm{CO}$ adsorption. The breakthrough curves of the formaldehyde adsorption, acetone adsorption and propylene glycol adsorption versus adsorption time at different adsorption temperatures on cellulose acetate stick are shown in Figures 9a, $9 b$, and 9c, respectively. The result revealed that the formaldehyde, acetone, and propylene glycol adsorption characteristics followed the same trend as CO adsorption. Therefore, it can be concluded that the adsorption capacities of cellulose acetate stick for all the typical smoke stream substances are all dependent on the adsorption time and temperature. At the same adsorption temperature, the adsorption capacities of smoke substances increased with the adsorption time until the surface adsorption of cellulose acetate stick was saturated. The relatively higher speed of adsorption in the beginning of the adsorption process can be connected with the high amount of free active sites which can be filled with smoke substances. Meanwhile, the adsorption characteristics of all these typical smoke stream substances fit well with the Freundlich model (Oh et al., 2020). It is worth noting that the adsorption capacity of the cellulose acetate stick for different type of typical smoke substances is different because of the differences in chemical properties and molecular size of smoke substances (Xiao et al., 2021). In particular, the propylene glycol adsorption affinity of cellulose acetate stick is greatest 
among the smoke substances with the lowest adsorption equilibrium time point due to the closest polarity to cellulose acetate (Davydov and Posrednik, 2020). Based on the

266 obtained data, it could be concluded that the absorption abilities of smoke substances

267 decrease with the adsorption temperature at the same adsorption time, as the high 268 temperature is not conductive to the absorption of smoke substances on the surface of 269 cellulose acetate stick.

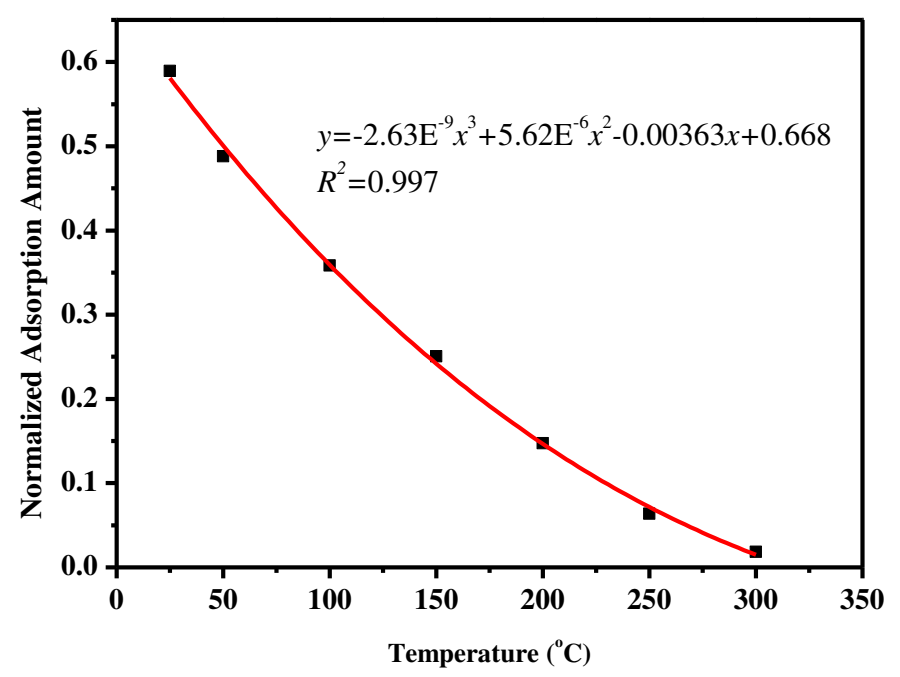

271 Figure 10. The fitting curve for CO adsorption ability versus adsorption temperature 272 after $\mathrm{CO}$ adsorption saturation, insertion is the fitted polynomial equation. In order to observe the adsorption capacity of cellulose acetate stick for typical

274 smoke stream substances changes with the temperature/space coordinate intuitively, the 275 transformation of the data of the breakthrough curves is required. Take the CO 276 adsorption as an example, the experimental results of $\mathrm{CO}$ adsorption presented in this work allowed for a mathematical model to be established [Equation.3], describing the 278 normalized $\mathrm{CO}$ adsorption amount versus the different temperatures. The 
corresponding mathematical model was obtained by fitting the data in Figure 10 with the equation as follows:

$$
\mathrm{CO}_{\text {adsorption }}=-2.63 E^{-9} \times T^{3}+5.62 E^{-6} \times T^{2}-0.00363 T+0.668 \quad \text { (Equation.3) }
$$

The reason for acquiring and mathematically modeling the data presented in Figure 10 is that it can be used to predict and calibrate the $\mathrm{CO}$ adsorption ability at any temperature in the range of $25-930^{\circ} \mathrm{C}$. Therefore, this mathematical model is significant to predict $\mathrm{CO}$ dynamic variations in the whole cellulose acetate stick. Specifically, combined the above mathematical model [Equation.3] with the temperature gradient model equation [such as Equation.1], we can easily get the $\mathrm{CO}$ adsorption characteristics along the radial direction in the 2D cross-sections; take the 2D crosssection at $x=10 \mathrm{~mm}$ for example. The fitting curves of normalized $\mathrm{CO}$ adsorption for the $2 \mathrm{D}$ section at $x=10 \mathrm{~mm}$ acquired by the mathematical treatment with Figures $5 \mathrm{~b}$ and 10 are shown in the Figure $11 \mathrm{a}$, the corresponding equations of the curves that fitted are as followed:

$\mathrm{CO}_{\text {adsorption }}=0(0 \leq r \leq 1.6)$ (Equation.4) $\mathrm{CO}_{\text {adsorption }}=-0.0874 r^{3}+0.716 r^{2}-1.572 r+1.005(1.6<r \leq 4) \quad($ Equation.5)

After the $\mathrm{CO}$ adsorption characteristics of all the 2D cross-sections were identified, we could calculate the average $\mathrm{CO}$ adsorption ability at different $2 \mathrm{D}$ cross-sections by the integral method. Then, the information on the average $\mathrm{CO}$ adsorption along the different $x$-axis was acquired (Figure 11b) and fitted in the same way as the Equation. 2: 

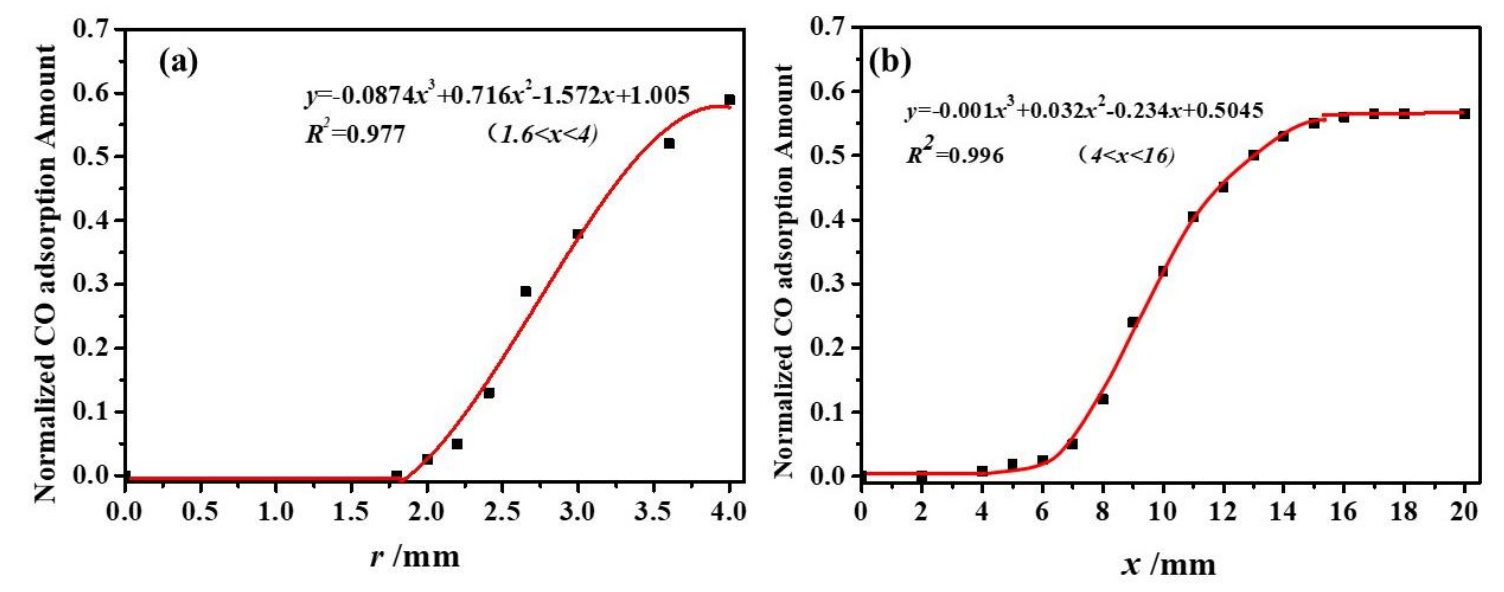

304 Figure 11. The fitting curve for $\mathrm{CO}$ adsorption ability, the insertions are the fitted 305 polynomial equations: (a) along $r$-axis at $x=10 \mathrm{~mm}$; (b) along $x$-axis stick could be roughly calculated by the integration, yet, it was not possible to

308 intuitively analyze the dynamic alteration of $\mathrm{CO}$ adsorption In order to solve this 309 problem, Figure 12 shows the 3D model of the normalized CO adsorption of cellulose acetate stick versus the spatial coordinates. The normalized $\mathrm{CO}$ adsorption can be

311 observed at any point in the cellulose acetate stick. Almost no CO adsorption is

312 observed in the area near the tobacco burning side due to their higher temperature

$313\left(>300{ }^{\circ} \mathrm{C}\right)$. Then the $\mathrm{CO}$ adsorption ability gradually increased both along the $x$-axis

314 and $r$-axis until it was saturated as the temperature gradually reduced. Compared with

315 Figure $11 \mathrm{~b}$, we could exactly calculate the total amount of adsorbed $\mathrm{CO}$ in whole 
cellulose acetate stick with the 3D adsorption model (Figure 12) by the integral method at any time point. Based on this, the dynamic distributions of the typical smoke stream

318 substances could also be modeled with the normalized adsorption amount of versus the

319 time. With it, the amount of typical smoke stream substances released by cigarettes after

320 the interception of cellulose acetate stick could be controlled. The CO-TPD experiments also detailed the adsorption and retention characteristics (Figure S2).

Before desorption measurement, $\mathrm{CO}$ adsorption was conducted at $50{ }^{\circ} \mathrm{C}$. An evident pear appeared in the pattern, which could be assigned to the characteristic peak of physical adsorption of $\mathrm{CO}$. To some extent, the effective desorption indicated that the stick was effective in retaining $\mathrm{CO}$ at low temperatures.

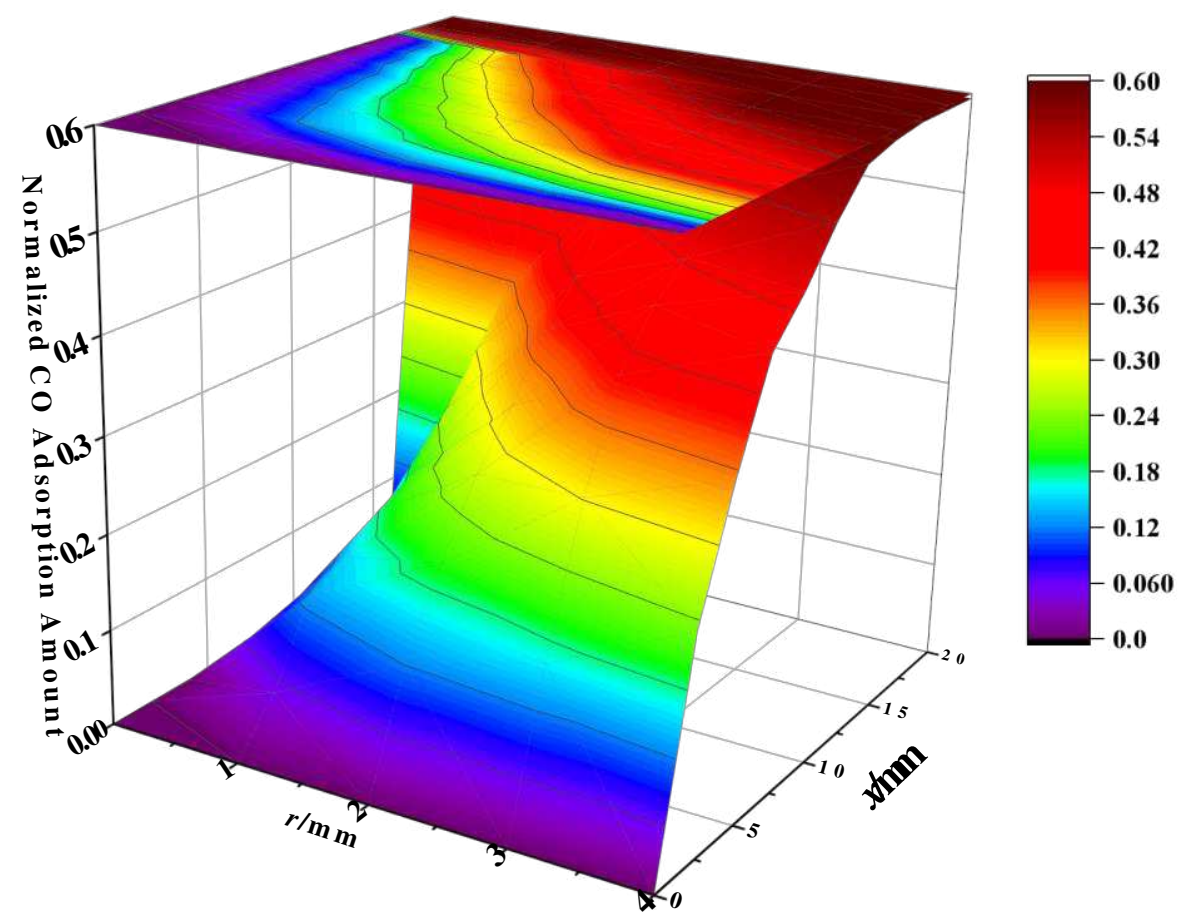

Figure 12.3D model of the Normalized CO adsorption amount of cellulose acetate stick versus different $x$ axis and $r$ axis 


\section{Conclusions}

A combined in-situ DRIFTS and the infrared thermal imaging method were established and applied to investigate the adsorption of $\mathrm{CO}$ and typical low-carbon aldehydes, formaldehyde, propylene glycol, and acetone, on cellulose acetate stick. The 3D temperature distribution with the fitting curves of temperature versus $x$-axis and $r$ axis was obtained by the mathematic fitting with the infrared thermal images results for confirming the adsorption temperature range, which was used to perform in-situ DRIFTS experiment in accurate temperature conditions. The adsorption capacities of cellulose acetate stick to these typical smoke stream substances were correlated with the adsorption time and temperature, and the corresponding models equations in cellulose acetate stick were established. The adsorption ability of the cellulose acetate stick for the adsorption of different smoke substances was different because of differences in chemical properties and molecular size of smoke substances. The propylene glycol adsorption was strongest among smoke stream substances. By combining the $3 \mathrm{D}$ temperature distribution model with the adsorption model equations, the 3D model of adsorption capacity versus the spatial coordinates was established for intuitively illustrating the dynamic distributions of the typical smoke stream. The information obtained in this work is useful for guiding analytical pyrolysis studies aimed at assessing precursor-smoke toxicant relationships and the fate of tobacco ingredients added to cigarettes. 
351 This study was supported by the National Natural Science Foundation of China 352 (U1832165 and 21902001), Anhui Provincial Natural Science Foundation 353 (2008085QB85), Key Research and Development Program of Anhui Province 354 (202004a05020015 and 006233172019), and Higher Education Natural Science 355 Foundation of Anhui Province (K120433040).

356

\section{Declarations}

358 Conflict of interest Authors declare no conflicts or competing interests. 


\section{References}

Adam, T., Mitschke, S., Streibel, T., Baker, R. R., and Zimmermann, R. (2006). Quantitative Puffby-Puff-Resolved Characterization of Selected Toxic Compounds in Cigarette Mainstream Smoke. Chem Res Toxicol 19, 511-520.

Alalwan, H., and Alminshid, A. (2020). An in-situ DRIFTS study of acetone adsorption mechanism on $\mathrm{TiO}_{2}$ nanoparticles. Spectrochimica Acta Part A: Molecular and Biomolecular Spectroscopy $229,117990$.

Boue, S., Goedertier, D., Hoeng, J., Kuczaj, A., Majeed, S., Mathis, C., May, A., Phillips, B., Peitsch, M., Radtke, F., et al. (2020). State-of-the-art methods and devices for the generation, exposure, and collection of aerosols from heat-not-burn tobacco products. Toxicology Research and Application 4. https://doi.org/10.1177/2397847319897869

Chau, N., Nghiem, T., Doan, H., Hoang Nguyen Do, N., Tran, V., Nguyen, S., and Le, P. (2020). Advanced Fabrication and Applications of Cellulose Acetate Aerogels from Cigarette Butts. MATERIALS TRANSACTIONS 61, 1550-1554.

Davydov, S., and Posrednik, O. (2020). Adsorption of Polar Molecules on a Solid Substrate. Mechanics of Solids 55, 90-93.

Dong, W., Qian, F., Li, Q., Tang, G., Xiang, T., Chun, T., Lu, J., Han, Y., Xia, Y., and Hu, J. (2021). Fabrication of superhydrophobic PET filter material with fluorinated $\mathrm{SiO}_{2}$ nanoparticles via simple sol-gel process. J Sol-Gel Sci Technol 98, 224-237.

Du, W., Jianhui, W., Bing, P., Xiaobin, Z., Xie, F., Huimin, L., and Kejun, Z. (2015). An Improved Theoretical Model of Cigarette Smoke Filtration across Mono-Segment Cellulose Acetate Filters. Beiträge zur Tabakforschung/Contributions to Tobacco Research 26, 232-240.

Dwyer, R. W., and Abel, S. G. (2015). The Efficiencies of Cellulose Acetate Filters. Beiträge zur Tabakforschung / Contributions to Tobacco Research 13, 243-253.

Dzara, M. J., Artyushkova, K., Shulda, S., Strand, M. B., Ngo, C., Crumlin, E. J., Gennett, T., and Pylypenko, S. (2019). Characterization of Complex Interactions at the Gas-Solid Interface with in Situ Spectroscopy: The Case of Nitrogen-Functionalized Carbon. J Phys Chem C 123, 90749086.

Fei, T., Chen, M., Zheng, S., Zhang, Y., Wang, X., Fu, J., Wu, D., and Tong, Y. (2014). Sectional 
retaining efficiency of cellulose acetate filter to major phenols in mainstream cigarette smoke. Tobacco Science and Technology, 56-60.

Feng, S. A., Huang, T. S., Zou, K. X., Li, Z. H., Zhou, J., Wei, K., and Bai, J. F. (2013). Study on the selective adsorption of low-molecular aldehydes and ketones from cigarette smoke using mesoporous alumina. Gongneng Cailiao/Journal of Functional Materials 44, 63-66.

Jiang, Z., Ding, X., Fang, T., Huang, H., Zhou, W., and Sun, Q. (2018). Study on heat transfer process of a heat not burn tobacco product flow field. Journal of Physics: Conference Series 1064, 012011.

Jia, T., Wang, H., Chen, D., Hou, M., Wu, C., Tu, M., Jiang, Y., and Zhang, S. (2017). 3D Temperature Distribution Model Based on Thermal Infrared Image. Journal of Sensors, 4815021. https://doi.org/10.1155/2017/4815021

401

Li, B., Pang, H. R., Zhao, L. C., Wang, B., Liu, C., McAdam, K. G., and Luo, D. S. (2014). Quantifying Gas-Phase Temperature inside a Burning Cigarette. Industrial \& Engineering Chemistry Research 53, 7810-7820.

Li, B., Zhao, L. C., Wang, L., Liu, C., McAdam, K. G., and Wang, B. (2016). Gas-phase pressure and flow velocity fields inside a burning cigarette during a puff. Thermochim Acta 623, 22-28.

Li, C., Li, E. x., Zhang, J., Tao, Y., Zhang, C., Shen, X., Liu, Y., and Qin, Y. (2018). Analysis of crotonaldehyde puff-by-puff release in mainstream cigarette smoke under various smoking regimens by high-performance liquid chromatography with the modified QuEChERS method. Royal Society Open Science 5, 172003.

Li, C., Zhang, J., Li, E. X., Qin, Y., Zhang, C., Li, Z., and Jiang, L. (2015). Puff-by-puff release characteristics of benzo [a] pyrene in mainstream cigarette smoke under various smoking regimes. $48,58-63$.

Li, H., Sun, J., Li, G., Wu, D., and Wang, Y. (2020). Real-time monitoring of surface acetone enolization and aldolization. Catalysis Science \& Technology 10, 935-939.

Ma, L. (2021). Are E-Cigarettes Safer than Cigarette Smoking? Biomedical Journal of Scientific \& Technical Research 33, 26224-26226.

Meunier, F. C. (2016). Pitfalls and benefits of in situ and operando diffuse reflectance FT-IR spectroscopy (DRIFTS) applied to catalytic reactions. Reaction Chemistry \& Engineering 1 , 
134-141.

Oh, H., Beum, H. T., Yoon, Y.-S., Kim, J., Han, Y., Kim, J., Lee, I.-B., Lee, S.-Y., and Han, S. S. (2020). Experiment and Modeling of Adsorption of CO from Blast Furnace Gas onto $\mathrm{CuCl} /$ Boehmite. Industrial \& Engineering Chemistry Research 59, 12176-12185.

Pasupathi, P., G, B., Yy, R., and J, F. (2009). Cigarette smoking and the health risk-Review article. Diabetes \& Metabolic Syndrome: Clinical Research \& Reviews 3, 120-127.

Pauwels, C. G. G. M., Klerx, W. N. M., Pennings, J. L. A., Boots, A. W., van Schooten, F. J., Opperhuizen, A., and Talhout, R. (2018). Cigarette Filter Ventilation and Smoking Protocol Influence Aldehyde Smoke Yields. Chem Res Toxicol 31, 462-471.

Proaño, L., Tello, E., Arellano-Trevino, M. A., Wang, S., Farrauto, R. J., and Cobo, M. (2019). Insitu DRIFTS study of two-step $\mathrm{CO}_{2}$ capture and catalytic methanation over $\mathrm{Ru}$, "Na $\mathrm{Na}_{2} \mathrm{O}$ " $/ \mathrm{Al}_{2} \mathrm{O}_{3}$ Dual Functional Material. Appl Surf Sci 479, 25-30.

Pham Xuan, R., Xiong, Y., Brietzke, A., and Marker, S. (2021). Thermal infrared imaging based facial temperature in comparison to ear temperature during a real-driving scenario. Journal of Thermal Biology 96, 102806.

Roehl, K., Becker, S., Fuhrmeister, C., Teuscher, N., Füting, M., and Heilmann, A. (2009). New, non-invasive thermographic examination of body surface temperature on tetraplegic and paraplegic patients, as a supplement to existing diagnostic measures. Spinal Cord 47, 492-495.

Shi, X., Wang, Y., Shan, Y., Yu, Y., and He, H. (2020). Investigation of the common intermediates over Fe-ZSM-5 in $\mathrm{NH}_{3}$-SCR reaction at low temperature by in situ DRIFTS. Journal of Environmental Sciences 94, 32-39.

Starek, A., and Podolak, I. (2009). Carcinogenic effect of tobacco smoke. Roczniki Państwowego Zakładu Higieny 60, 299-310.

Tofan-Lazar, J., Situm, A., and Al-Abadleh, H. A. (2013). DRIFTS Studies on the Role of Surface Water in Stabilizing Catechol-Iron(III) Complexes at the Gas/Solid Interface. J Phys Chem A 117, 10368-10380.

Wang, L., Wang, Z., Cheng, X., Zhang, M., Qin, Y., and Ma, C. (2017). In situ DRIFTS study of the $\mathrm{NO}+\mathrm{CO}$ reaction on $\mathrm{Fe}-\mathrm{Co}$ binary metal oxides over activated semi-coke supports. RSC Advances 7, 7695-7710. 
447 Xiao, Y., Yang, H., Bu, X., and Feng, P. (2021). ZIF-8 Derived Carbon Materials with $448 \quad$ Multifunctional Selective Adsorption Abilities. Carbon 176.

449 Yang, L., Liu, L., Liu, H., Yi, L., Gu, X., Liu, H., and Wang, H. (2021). Adsorption behavior of $\mathrm{CO}_{2}$ 450 in magnesite micro-pores at high temperature and pressure. Geoscience Frontiers 12, 991-999. 451 Zhang, Q., Fang, C., Cheng, Y., Chen, J., Huang, Z., and Han, H. (2020). Construction and properties 452 of cellulose diacetate film derived from waste cigarette filters. Cellulose 27, 8899-8907.

453 Zhong, J., Zeng, Y., Zhang, M., Feng, W., Xiao, D., Wu, J., Chen, P., Fu, M., and Ye, D. (2020). 454 Toluene oxidation process and proper mechanism over $\mathrm{Co}_{3} \mathrm{O}_{4}$ nanotubes: Investigation through 455 in -situ DRIFTS combined with PTR-TOF-MS and quasi in -situ XPS. Chem Eng J 397, 1-12. $456 \quad$ https://doi.org/10.1016/j.cej.2020.125375 


\section{Supplementary Files}

This is a list of supplementary files associated with this preprint. Click to download.

- Slsubmission.docx 